УДК 519.6

MSC $65 \mathrm{~K} 10$

\title{
OPTIMAL CONTROL OF A TWO-DIMENSIONAL RICHARDS-KLUTE EQUATION
}

\author{
ANDRII TYMOSHENKO
}

Faculty of Computer Science and Cybernetics, Taras Shevchenko National University of Kyiv, Kyiv, Ukraine, E-mail: inna-andry@ukr.net

\section{ОПТИМАЛЬНЕ КЕРУВАННЯ ДВОВИМІРНИМ РІВНЯННЯМ РІЧАРДСА-КЛЮТА}

\section{А. А. Тимошенко}

Факультет комп'ютерних наук та кібернетики, Київський національний університет імени Тараса Шевченко, Київ, Україна, E-mail: inna-andry@ukr.net

ABstract. This article demonstrates an approach to optimal control of humidity using point sources for the two-dimensional problem. The mathematical model is based on Richards-Klute equation. The desired humidity state at the last moment is set and the solution should reach it from the known initial state by optimal source power. The moisture is assumed incompressible, the temperature and external pressure are constant.

KEYWORDS: control, optimization, Richards-Klute equation.

АнотАція. У статті демонструється підхід до оптимального керування вологістю за допомогою точкових джерел для двовимірної задачі. Математична модель основана на рівнянні РічардсаКлюта. В останній момент часу задано бажаний розподіл вологості, до якого з відомого початкового стану за допомогою керування потужністю джерел має прямувати розв'язок. Рідина вважається нестискуваною, температура та зовнішній тиск є постійними.

КлЮчові словА: управління, оптимізація, рівняння РічардсаКлюта.

\section{ВСТУП}

Задачі переносу вологи у пористому середовищі класично базуються на законі Дарсі та його модифікаціях, рівняння Річардса є розповсюдженим для лінійних та нелінійних постановок задач [1]. Оскільки врахувати усі фізичні та хімічні фактори впливу на процес складно, вводиться ряд спрощень - рідину вважаємо нестискуваною, тиск та температуру постійними. Задача моделювання вологості є достатньо поширеною. Аналітичні результати розподілу вологи при впливі кількох точкових джерел та пропозиція щодо лінеаризації деяких типів нелінійних рівнянь наведені у [2]. 
Деякі алгоритми пошуку наближеного розв'язку нелінійних рівнянь, питання існування та єдиності розглянуто у [3]. В залежності від підходу до моделювання, початкова задача може бути нелінійною або квазілінійною $[4,5]$. Емпіричні залежності для процесу переносу вологи у кількох типів грунтів проаналізовано в [6]. До лінеаризованого рівняння після його дискретизації за часом та простом, застосовується позмінно-трикутний метод, наведений у [7]. За цим методом відбувається перехід до системи лінійних алгебраїчних рівнянь з урахуванням граничних умов, що залежить від двох координат у просторі та моменту часу. Для розв'язання даної системи використовується метод Гаусса або метод Якобі [8]. Часова та просторова дискретизація для рівняння Річардса досліджена у [9-11]. Задача оптимального керування процесом переносу вологи все ще є актуальною. Питанням існування та єдиності розв'язку задачі оптимізації для лінійних систем та деяких нелінійних систем досліджено у [12-15].

Дана робота використовує метод оптимізації, розглянутий у [14]. Початкове наближення оптимальної потужності джерела шукається як сума різниць квадратів між значеннями бажаного стану у кінцевий момент часу та аналогічними значеннями, отриманими у результаті керування. Щодо спряжених операторів, для побудови спряженого рівняння використовуються результати, наведені у [16-20].

Таким чином, дана робота поєднує лінеаризацію початкової постановки задачі, перехід до системи лінійних алгебраїчних рівнять та оптимізацію потужності джерела.

\section{1. ЗАГАЛЬНА ПОСТАНОВКА ЗАДАЧІ}

Процес моделюється згідно [1]

$$
\frac{\partial \nu}{\partial t}=\operatorname{div}(\operatorname{Kgrad} \Psi)-\frac{\partial K}{\partial z} .
$$

Відповідно, введено позначення $\Psi$ - гідравлічний потенціал рідини, $K-$ водопроникність, $\nu$ - вологість середовища, $t$ - час, $z$ - вертикальна координата простору. Дане рівняння може описувати як однорідне, так і неоднорідне середовище.

У випадку, коли $K$ та $\Psi$ залежать лише від $\nu$, то зручно ввести дифузійність

$$
D(\nu)=K(\nu) \frac{d \Psi}{d \nu}
$$

та підставивши $\dddot{11}$, отримати

$$
\frac{\partial \nu}{\partial t}=\operatorname{div}(D \operatorname{grad} \nu)-\frac{d K}{d \nu} \frac{\partial \nu}{\partial z} .
$$

Функціональні залежності $K(\nu), \Psi(\nu), D(\nu)$ можуть бути нелінійними, тому вводиться потенціал Кірхгофа

$$
\Theta=\int_{\nu_{1}}^{\nu} D(\nu) d \nu .
$$


Тоді можна сформулювати рівняння у вигляді

$$
\frac{1}{D(\nu)} \frac{\partial \Theta}{\partial t}=\operatorname{div} \operatorname{grad} \Theta-\frac{1}{K(\nu)} \frac{d K}{d \nu} \frac{\partial \nu}{\partial z} .
$$

\section{2. ЛІНІЙНА ПОСТАНОВКА ЗАДАЧІ}

Скористаємось результатами Новосельського [2], де досліджено вплив зосереджених джерел на вологість грунту.

Розглянемо задачу переносу вологи у вертикальній площині на обмеженій області

$$
\begin{gathered}
\frac{\partial \omega}{\partial t}=\frac{\partial}{\partial x}\left[K_{x}(\omega) \frac{\partial H}{\partial x}\right]+\frac{\partial}{\partial z}\left[K_{z}(\omega) \frac{\partial H}{\partial z}\right]+\sum_{j=1}^{N} Q_{j}(t) \delta\left(x-x_{j}\right) \delta\left(z-z_{j}\right), \\
z=\left\{0, L_{2}\right\}, \quad \frac{\partial \omega}{\partial z}=0, \\
x=\left\{0, L_{1}\right\}, \quad \frac{\partial \omega}{\partial x}=0, \\
\omega(x, z, 0)=\varphi(x, z), \quad(x, z) \in \bar{\Omega},
\end{gathered}
$$

у якій позначено $H=\Psi(\omega)-z-$ напор, $D_{z}(\omega)=K_{z}(\omega) \frac{d \psi}{d \omega}-$ дифузійність вздовж осі $z, \Omega_{0}=\left[(x, z): 0<x<L_{1}, 0<z<L_{2}\right], z=z_{0}$ - рівень поверхні грунту. Аналогічно до [17], визначимо проникність за кожним виміром

$$
K_{x}(\omega)=k_{1} k(\omega), K_{y}(\omega)=k_{2} k(\omega), K_{z}(\omega)=k_{3} k(\omega),
$$

де $k_{1}, k_{2}, k_{3}$ - фільтраційні коефіцієнти відповідно до головних вісей $O x$, $O y, O z, k(\omega)$ - функція вологості грунту. Для переходу до безрозмірної задачі використовуємо коефіцієнти: $\tilde{x}=\frac{\beta_{1}}{L_{1}} x, \tilde{z}=\frac{\beta_{3}}{L_{2}} z, \tau=\frac{\alpha t}{T}, \beta_{3}=0.5 \ell$, $\beta_{1}=\sqrt{\frac{k_{3}}{k_{1}}} \beta_{3}, \beta_{2}=\sqrt{\frac{k_{3}}{k_{1}}} \beta_{3}, \alpha=\left\langle D_{z}\right\rangle \beta_{2}^{2}$.

Перетворення Кірхгофа подається як

$$
\Theta=\frac{4 \pi \sqrt{k_{1} k_{2}}}{Q^{*} k_{3} \beta_{3}} \int_{0}^{\omega} D_{z}(\omega) d \omega,
$$

Тоді лінеаризована та обезрозмірена постановка задачі матиме вигляд:

$$
\begin{gathered}
L \Theta=\frac{\partial \Theta}{\partial \tau}-\Delta \Theta+2 \frac{\partial \Theta}{\partial \tilde{z}}=4 \pi \sum_{j=1}^{N} q_{j}(\tau) \delta\left(\tilde{x}-\tilde{x}_{j}\right) \delta\left(\tilde{z}-\tilde{z}_{j}\right), \\
(\tilde{x}, \tilde{z}, \tau) \in \Omega \times(0, T), \\
\tilde{z}=\{0,1\}, \quad \frac{\partial \Omega}{\partial \tilde{z}}=0, \\
\tilde{x}=\{0,1\}, \quad \frac{\partial \Theta}{\partial \tilde{x}}=0, \\
(\tilde{x}, \tilde{z}, \tau) \in \Gamma \times[0,1) .
\end{gathered}
$$


Початкові умови задано у вигляді

$$
\Theta(\tilde{x}, \tilde{z}, 0)=\varphi_{1}(\tilde{x}, \tilde{z}), \quad(\tilde{x}, \tilde{z}) \in \bar{\Omega},
$$

згідно позначень, $q_{j}=\frac{Q_{j}}{Q^{*}}, Q^{*}-$ векторний параметричний масштабний множник, $\Omega, \Gamma-$ безрозмірні аналоги $\Omega_{0}, \Gamma_{0},\left\langle D_{z}\right\rangle$ - середне значення $D_{z}(\omega), \varphi_{1}=\Theta(\varphi)$.

На перехід до лінійної задачі накладено умови [2]:

- лінійність зв'язку між $\Theta(\omega)$ та $K_{z}(\omega): D_{z}^{-1}(\omega) \frac{d K_{x}(\omega)}{d \omega}=\ell=$ const;

- для лінеаризації використовується заміна

$$
\frac{\partial \omega}{\partial t}=\frac{k_{3} \beta_{3} Q^{*}}{4 \pi \sqrt{ } k_{1} k_{2}} \frac{1}{D_{z}(\omega)} \frac{\partial \Theta}{\partial t} \simeq \frac{k_{3} \beta_{3}^{3} Q^{*}}{4 \pi \sqrt{ } k_{1} k_{2}} \frac{\partial \Theta}{\partial \tau} .
$$

Такий підхід дозволяє звести частину нелінійних задач до лінеаризованих.

\section{3. УПРАВЛІННЯ ТА ОПТИМІЗАЦІЯ}

Задача керування формулюється аналогічно [14] щоб скористатись доведеними існуванням та єдиністю розв'язку.

Позначимо $r_{\beta}$ - точки знаходження джерел деякої невідомої потужності $q_{\beta}(t)$. Концентрація рідини $\phi(x, z, t)$ представлена неперервною функцією. Задача полягає у знаходженні $q_{\beta}(t), \beta=\overline{1, p}$ згідно умови мінімізації модуля різниці цільового значення вологості $\varphi(x, z, t)$ та фактичними показниками точкових вимірювальних пристроїв $u(x, z, t)$.

Будемо вважати оптимальне управління приналежним гільбертовому простору з визначеним нижче скалярним добутком

$$
\langle X, Y\rangle=\sum_{\beta=1}^{p} \int_{0}^{T} x_{\beta}(t) y_{\beta}(t) d t
$$

Оптимізаційний функціонал запишемо у вигляді

$$
J_{\alpha}(Q)=\sum_{m=1}^{m} \int_{0}^{T} \int_{\Omega}(\phi(t)-u(t, x, z) d x d z)^{2} d t+\alpha\|Q\|^{2},
$$

в якому $\alpha>0$ - регуляризаційний параметр, що враховує похибки. Тоді поставлена оптимізаційна задача управління зводиться до вигляду

$$
J_{\alpha}\left(Q^{*}\right)=\min _{Q \in H} J_{\alpha}(Q) .
$$

Варіаційний алгоритм [14] містить пряму задачу, спряжену задачу та крок уточнення:

- пряма задача

$$
\frac{\partial \Theta^{(k)}}{\partial \tau}+L \Theta^{(k)}=f^{(k)}, \quad 0<t \leq T, \quad \Theta^{(k)}(0)=g(x) ;
$$


- спряжена задача

$$
-\frac{\partial \psi^{(k)}}{\partial \tau}+L^{*} \psi^{(k)}=2\left(\Theta^{(k)}-\phi(t)\right), \quad 0<t \leq T, \quad \psi^{(k)}(T)=0
$$

- пошук наступного наближення значення оптимальної потужності

$$
\frac{Q^{(k+1)}-Q^{(k)}}{\tau_{k+1}}+\Psi^{(k)}+\alpha Q^{(k)}=0, k=0,1, \ldots
$$

Покладемо $0<\alpha<\min \left(\tau, h^{2}\right)$.

Таким чином, задача оптимізації потребує знаходження спряжених операторів [16-20].

\section{4. МОДЕЛЬНА ЗАДАЧА}

Розглядається двовимірна область, на яку діє джерело постійної інтенсивності та вологість задана у початковий момент часу. Цільову вологість представляє досяжна неперервна функція. Оптимізаційний підхід полягає у пошуку інтенсивності, за якої досягається максимально схожа вологість у кінці часового проміжку.

Досліджується двовимірна задача вигляду

$$
L \Theta=\frac{\partial \Theta}{\partial \tau}-\frac{\partial^{2} \Theta}{\partial \tilde{z}^{2}}-\frac{\partial^{2} \Theta}{\partial \tilde{x}^{2}}+2 \frac{\partial \Theta}{\partial \tilde{z}}=-f(\tilde{x}, \tilde{z}, \tau)+4 \pi q_{j} \delta\left(\tilde{z}-\tilde{z}_{j}\right) \delta\left(\tilde{x}-\tilde{x}_{j}\right) .
$$

Коефіцієнти водопроникності взяті рівними 0.1 за всіма осями, на нижній границі області задано умову непроникності, перетворення Кірхгофа подається у вигляді

$$
\Theta=\frac{4 \pi \sqrt{k_{1} k_{2}}}{Q^{*} k_{3} \beta_{3}}\left(-2+2 e^{0.5 \omega}\right) .
$$

Часова дискретизація виконується з 10000 кроків у випадку явної та 100 кроками у випадку неявної схеми, а за простором $-h=\frac{1}{30}$. Для прямого рівняння запишемо згідно позмінно-трикутного методу [7] систему рівнянь вигляду:

$$
\Lambda(\tilde{x}, \tilde{z})=\Lambda_{1}(\tilde{x})+\Lambda_{2}(\tilde{z})=\frac{\partial \Theta}{\partial \tau}-\left(\varphi(\tilde{x}, \tilde{z})+\frac{1}{h} \varphi_{1}(\tilde{x})+\frac{1}{h} \varphi_{2}(\tilde{z})\right)
$$

3 урахуванням граничних умов, система в загальному вигляді розписується, як

$$
\begin{gathered}
\Lambda_{1}(\tilde{x})=\left\{\begin{array}{cc}
\frac{2}{h}\left(u_{x}\right), & \tilde{x}=0 ; \\
\left(u_{\bar{x}}\right)_{x}, & 0<\tilde{x}<1 ; \\
\frac{2}{h}\left(-u_{\bar{x}}\right), & \tilde{x}=1
\end{array}\right. \\
\Lambda_{2}(\tilde{z})=\left\{\begin{array}{cc}
\frac{2}{h}\left(u_{y}\right), & \tilde{z}=0 ; \\
\left(u_{\bar{y}}\right)_{y}-2 u_{\widehat{y}}, & 0<\tilde{z}<1 ; \\
\frac{2}{h}\left(-u_{\bar{y}}\right), & \tilde{z}=1
\end{array}\right.
\end{gathered}
$$

Тут $\widehat{y}$ позначає центральну різницеву похідну. 


$$
\varphi_{1}(\tilde{x})=\varphi_{2}(\tilde{z})=0
$$

Для кутового джерела покладемо

$$
\varphi(\tilde{x}, \tilde{z})=\left\{\begin{array}{cc}
4 \pi q, & \tilde{x}=0, \quad \tilde{z}=0 \\
0, & 0<\tilde{x} \leq 1, \quad 0<\tilde{z} \leq 1
\end{array}\right.
$$

Для джерела посередині верхньої границі маємо

$$
\varphi(\tilde{x}, \tilde{z})=\left\{\begin{array}{cc}
4 \pi q, & \tilde{x}=0.5, \quad \tilde{z}=0 \\
0, & \text { інакше }
\end{array}\right.
$$

У результаті значення $\Theta(\tilde{x}, \zeta)$ визначається з початкових та крайових умов, але вони залежать від $q$.

Початкове значення оптимальної потужності джерела обчислюється із задачі оптимізації для кінцевого моменту часу

$$
\min _{q}\left(\sum_{i, j=1}^{N}\left(\Theta\left(\tilde{x}_{i}, \tilde{z}_{j} ; q\right)-\phi\left(\tilde{x}_{i}, \tilde{z}_{j}\right)\right)^{2}+\alpha q^{2}\right) .
$$

Враховуючи, що $\Theta \geq 0$, у якості наближеного розв'язку можна обрати значення, отримане з рівності нулю похідної цього функціоналу.

Для зручності, виведемо співвідношення переходу від $\Theta$ до $\omega$ та навпаки. Отримаємо перетворення:

$$
\begin{gathered}
\Theta=\frac{4 \pi \sqrt{k_{1} k_{2}}}{Q^{*} k_{3} \beta_{3}} \int_{0}^{\omega} D_{z}(\omega) d \omega=\left[k 1=k 2=k 3, Q^{*}=1, \beta_{3}=0.5\right]= \\
=8 \pi\left(2 e^{0.5 \omega}-2\right)
\end{gathered}
$$

Тоді обернене перетворення має вигляд:

$$
\frac{\Theta}{16 \pi}=e^{0.5 \omega}-1 \Rightarrow \ln \left(\frac{\Theta}{16 \pi}+1\right)=0.5 \omega \Rightarrow \omega=2 \ln \left(\frac{\Theta}{16 \pi}+1\right)
$$

Введемо позначення $z^{*}=\frac{\tilde{z}_{i}}{h}, x^{*}=\frac{\tilde{x}_{i}}{h}$. Цільову функцію вологості задамо як результат моделювання при потужності 10. Ітераційний пошук оптимальної потужності виконується починаючи зі значення, близького до нуля.

Для цієї функції відхилення знайденої потужності від оптимальної складає приблизно 2 відсотків.

Графіки відхилень та ізолінії $\Theta$ для випадків кутового джерела та джерела посередині границі $z=0$ наведено нижче (Рис. $1,2,3,4$ ). 


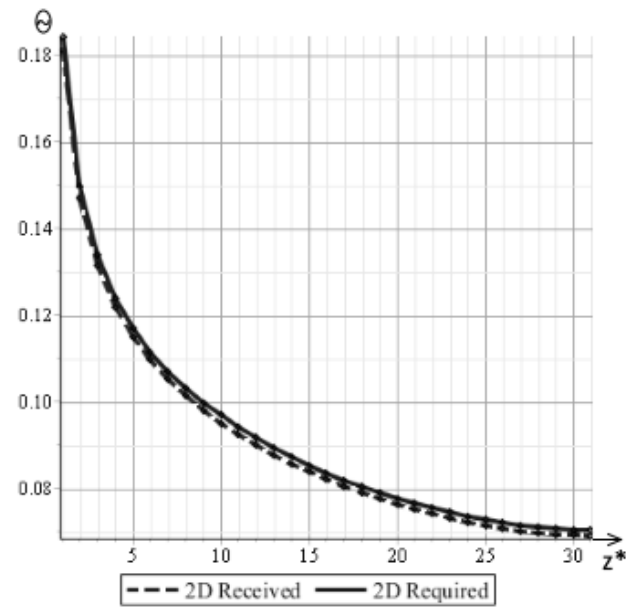

Рис. 1.

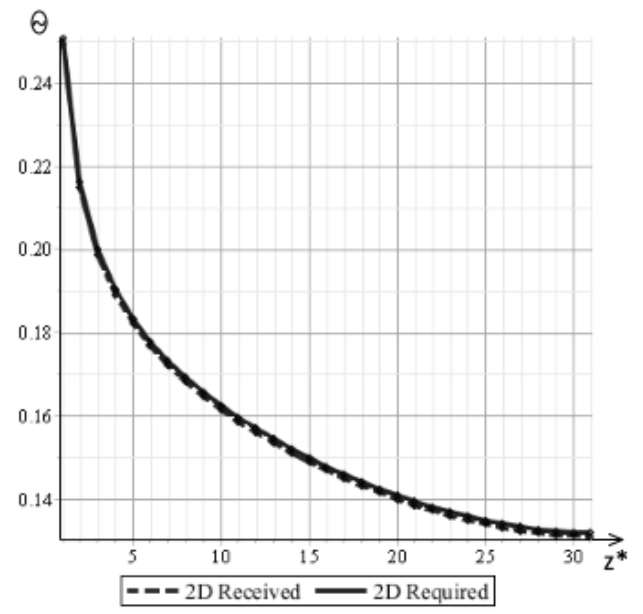

Рис. 2. 


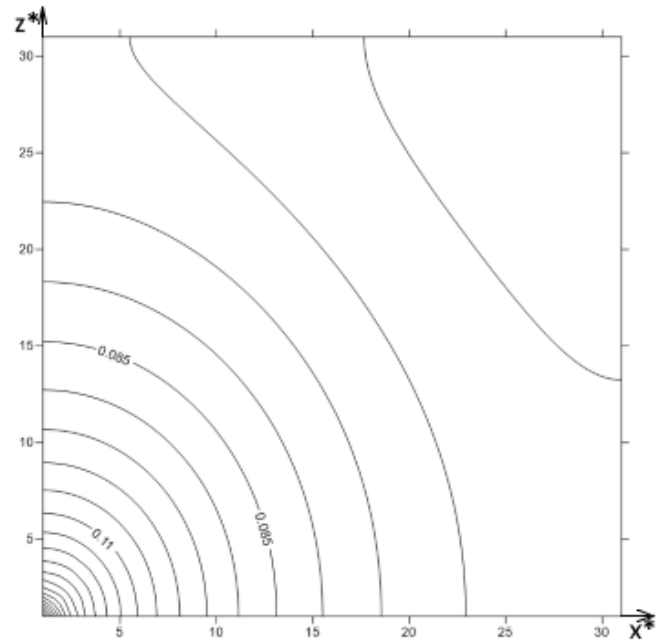

Рис. 3.

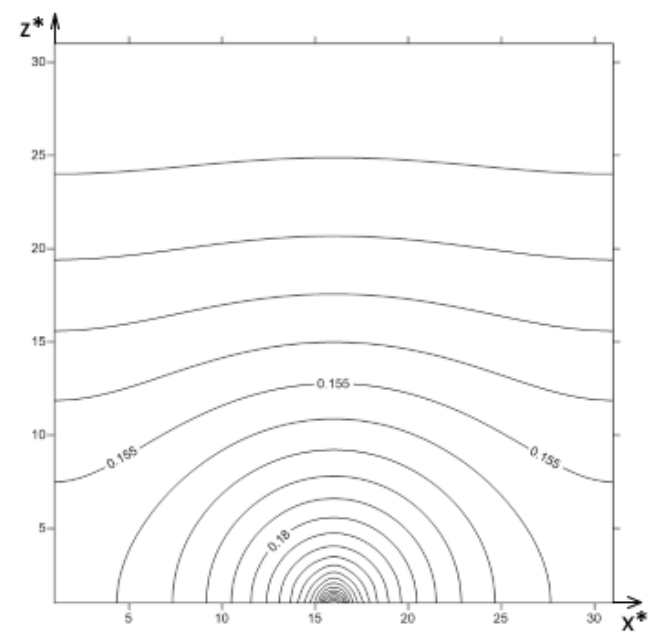

Рис. 4. 
Таким чином, для перевірки точності алгоритму цільова функція має бути досяжною, а початкове наближення потужності пришвидшуе роботу методу.

\section{Висновки}

При виконанні умов переходу, початкову задачу можна звести до лінеаризованої за допомогою перетворення Кірхгофа. Даний підхід також використовує позмінно-трикутний метод для запису системи лінійних рівнянь, розв'язавши яку можна отримати залежність вологості у кінцевий момент часу від потужності джерела у явному вигляді. Точність розв'язку задачі оптимізації залежить від досяжності цільової функції.

\section{ЛiтеРАТУРА}

1. Pullan A. J. The Quasilinear Approximation for Unsaturated Porous Media Flow. Water resources research. 1990. Vol. 26. No. 6. P. 1219-1234.

2. Novoselskiy S. N. Solution of some Boundary Value Problems of Moisture Transport with Irrigation Sources. Candidate thesis in Mathematics and Physics: 01.02.05. Kalinin Polytechnic Institute. Kalinin, 1981 (in Russian).

3. Gajevskiy H., Groger K., Zaharias K. Nonlinear operator equations and operator differential equations. Moscow: MIR. 336 p. (in Russian).

4. Van Genuchten M. A Closed-form Equation for Predicting the Hydraulic Conductivity of Unsaturated Soils. Soil Sci. Soc. Am. J. 1980. Vol. 44. P. 892-898.

5. Kashenko N. M. Processes of moisture transport in porous media. Vestnir Ros. gos. univ. im. I. Kanta. 2010. No. 10. P. 56-58 (in Russian).

6. Shatkovskiy A.P. Scientific Basics of Intensive Technologies for Drip Irrigation of Cultivated Plants Under Conditions of Ukrainian Steppes. Doctor thesis of Agricultural Sciences: 06.01.02. National Academy of Agrarian Sciences of Ukraine. Kyiv, 2016. 496 p.(in Ukrainian).

7. Samarskiy A. A., Nikolaev E. S. Methods for solving grid equations. Moscow: Science. 1978. 588 p.

8. Samarskiy A. A., Gulin A. V. Numerical methods. Moscow: Science. 1989. 432 p.

9. Arbogast T. An error analysis for Galerkin approximations to an equation of mixed elliptic-parabolic type. Technical Report TR90-33, Department of Computational and Applied Mathematics, Rice University. Houston, TX. 1990. 28 p.

10. Ginting V. Time integration techniques for Richards equation. Procedia Computer Science 9. 2012. P. 670-678. doi: https://doi.org/10.1016/j.procs.2012.04.072

11. Pop I. S. Error estimates for a time discretization method for the Richards' equation. Comput. Geosci. 6. 2002. P. 141-160.

12. Lions J.-L. Optimal control of systems, described by partial differential equations. Moscow: MIR. 1972. 416 p. (in Russian).

13. Kirk D. E. Optimal control theory. An Introduction. New Jersey: Dover Books on Electrical Engineering, 1971. 472 p. doi: https://doi.org/10.1002/aic.690170452

14. Lyashko S., Klyushin D., Semenov V., Shevchenko K. Identification of point contamination source in ground water. International Journal of Ecology and Development. Fall 2006. Vol. 5. No.6. P. 36-43.

15. Lyashko S. I. Generalized control of linear systems. Kyiv: Nauk. dumka. 1998. 470 p. (in Russian). 
16. Vabishchevich P. N. Numerical Solution of the Problem of the Identification of the Right-hand Side of a Parabolic Equation. Russian Math. Iz. VUZ. 2003. 47:1. P. 29-37. (in Russian).

17. Marchuk G. I. Conjugate equations and their application. Tr. IMM UrO RAN. 2006. Vol 12. No 1. P. 184-195. (in Russian).

18. Marchuk G. I., Shutyaev V. P. Conjugate equations and iterational algorythms in problems of variational data assimilation. Tr. IMM UrO RAN. 2011. Vol. 17. No. 2. P. 136-150. (in Russian).

19. Marchuk G. I. About some approaches to building conjugate operators in nonlinear tasks. Tr. MIAN. 1994. Vol. 203. P. 126-134. (in Russian).

20. Marchuk G. I. Construction of conjugate operators in nonlinear problems of mathematical physics. Matem. sb. 1998. Vol. 189. No 10, P. 75-88. (in Russian).

Надійшла: 08.03.2019 / Прийнята: 20.05.2019 